\title{
Assembly and Dynamics of Myofibrils
}

\author{
Joseph W. Sanger, Jushuo Wang, Yingli Fan, Jennifer White, and Jean M. Sanger
}

Department of Cell and Developmental Biology, SUNY Upstate Medical University, Syracuse, NY 13210, USA

Correspondence should be addressed to Joseph W. Sanger, sangerjo@upstate.edu

Received 31 December 2009; Accepted 15 March 2010

Academic Editor: Aikaterini Kontrogianni-Konstantopoulos

Copyright ( $) 2010$ Joseph W. Sanger et al. This is an open access article distributed under the Creative Commons Attribution License, which permits unrestricted use, distribution, and reproduction in any medium, provided the original work is properly cited.

\begin{abstract}
We review some of the problems in determining how myofibrils may be assembled and just as importantly how this contractile structure may be renewed by sarcomeric proteins moving between the sarcomere and the cytoplasm. We also address in this personal review the recent evidence that indicates that the assembly and dynamics of myofibrils are conserved whether the cells are analyzed in situ or in tissue culture conditions. We suggest that myofibrillogenesis is a fundamentally conserved process, comparable to protein synthesis, mitosis, or cytokinesis, whether examined in situ or in vitro.
\end{abstract}

\section{Introduction}

Myofibrils of striated muscle are characterized by groups of proteins arranged in contractile units, or sarcomeres, that consist of distinct subunits that extend in a repeating pattern along the length of the muscle cell. Although sarcomeres of cross-striated muscle vary among species in length and in some protein constituents, all have a similar subunit arrangement of three major components: thin filaments, thick filaments, and Z-bands that each forms from multiple interactions among the proteins that produce and control contraction (Figure 1). Vertebrate skeletal and cardiac sarcomeres at rest length are 2.5 microns in length whereas the sarcomeres of cross-striated muscles in the invertebrate world vary widely from one micron in a jellyfish [1] to 25 microns in the pharynx of a syllid worm [2]. A-band lengths of thick filaments and thin filament lengths vary in concert with sarcomere size: the shortest at 0.6 microns and 0.5 microns, respectively, in jellyfish, the longest at 20 microns and 12 microns, respectively, in the syllid worm, and 1.6 microns and 1.0 micron in vertebrate skeletal muscle [3]. In vertebrate ventricular muscles, the thin filament lengths are variable up to one micron [4].

Despite all that is known about the binding of myofibrillar proteins, one with one another in biochemical assays, much is unknown about the multiplicity of interactions that support myofibril formation and stability in the live cell. The importance of myofibrillar protein interactions in cardiac health has been reinforced by the realization that mutated sarcomeric proteins are involved in a growing number of cardiomyopathies $[9,10]$. Interactions of the major proteins responsible for force production in the thin and thick filament subunits have been described in detail [11]. Proteins of the Z-band, a region of the sarcomere where novel proteins and novel interactions of known proteins are still being discovered $[12,13]$, present a particularly challenging network of potential multiple interactions, many of which have been determined by biochemical methods not by methods inside the living cell (Figure 2). The Z-band functions as a scaffold that links the sarcomeric contractile units in series by anchoring the thin and titin filaments of adjacent sarcomeres. Z-bands also anchor the ends of myofibrils in specialized junctions, termed intercalated discs in cardiac muscle cells, and they link sarcomeres laterally to the cell membrane through costameric proteins $[14,15]$. The multifunctional nature of the Z-band is reflected in the variety of proteins that colocalize in this structure: channels, signaling molecules, enzymes, cytoskeletal filament complex that interacts with the cell membrane, and sarcomeric filaments essential for contraction (Figure 2) [11, 16, 17]. Two of the most surprising recent reports on new Z-band proteins concern (a) heat shock proteins in zebrafish skeletal muscles (reviewed in [12]) and (b) the protein, CLOCK, which is involved in circadian regulation in neonatal rat cardiomyocytes (reviewed in [13]). 

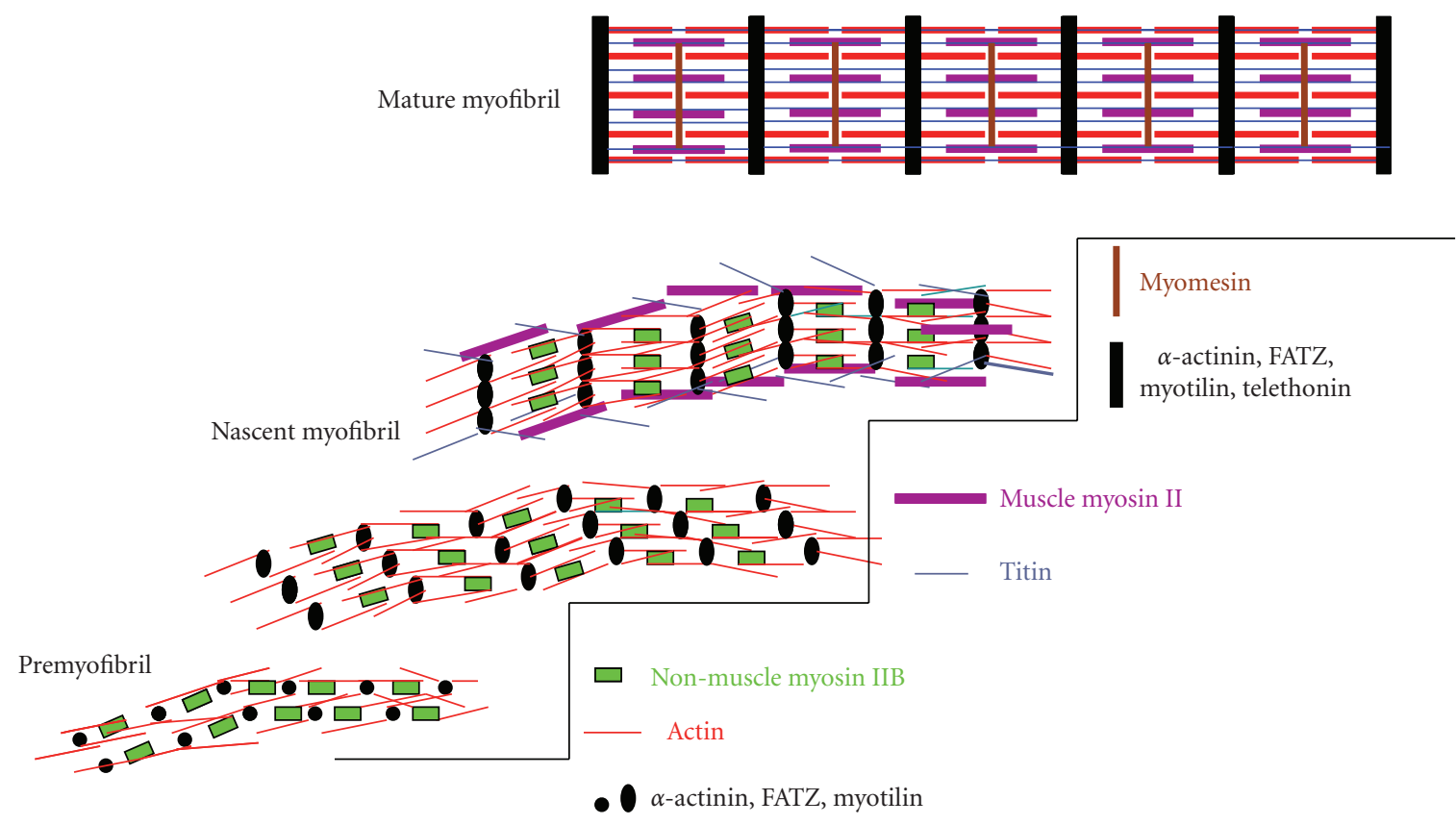

Figure 1: Premyofibril model of myofibrillogenesis. Assembly begins at the edges of muscle cells with premyofibrils composed of minisarcomeres (bounded by alpha-actinin containing z-bodies) with muscle actin and actin-binding proteins and the nonmuscle isoform of myosin II. Muscle myosin and titin are added and z-bodies align in register to form nascent myofibrils with beaded Z-bands. As mature myofibrils form, the beaded Z-bands gradually become linear, nonmuscle myosin is lost, and myosin-binding proteins (C-protein and myomesin) are incorporated into aligned A-bands [5-8]. Diagram modified from Stout et al. [8].

\section{Formation of Myofibrils}

As the list of myofibril proteins and their binding reactions grows, the assembly pathways that the interacting protein complexes follow to form myofibrils have increasing import for understanding the problems of aberrant formation and maintenance of myofibrils containing mutated sarcomeric proteins $[9,10,16,18]$. In the early 1900s, Heidenhain [19] examined fixed muscles in the light microscope and noted nonstriated fibers near the cell membrane of developing trout skeletal muscles before striated structures that we now know were Z-bands and A-bands were detected. Electron micrographs of forming chick muscle showed nonstriated fibers near the cell membrane, as well, before banded myofibrils appeared (see review by Sanger et al. [6]). Growth in A-band length to mature size was also observed with electron microscopy of embryonic fly muscle fixed at daily intervals. The first fibers observed were nonstriated with dense bodies but lacking thick filaments, succeeded by fibers with Z-bands and short A-bands and later by fibers with Abands of mature muscle length [20]. Striated sarcomeres in forming mite muscle that were analyzed with polarized light in the live animal increased over a 30-hour period from 2.2 microns to 4 microns in length, accompanied by an increase in A-band length from 1.4 microns to 3 micron [21].

The advent of probes for fluorescent detection of proteins inside cells allowed fibers in nonmuscle cells and embryonic muscle cells to be characterized. In nonmuscle cells many stress fibers, previously described as unstriated in $[22,23]$ were shown to be composed of repeating concentrations of alpha-actinin and nonmuscle myosin II distributed in alternating bands along overlapping actin filaments [24-28]. In a short presentation, a three-page report, published in a book describing the proceedings of short talks honoring the late John Marshall, Kulikowski and Manasek [29], described how embryonic chick cultured cardiomyocytes appeared when stained with muscle-specific myosin II antibodies after different periods of time in culture. They noted the appearance of nonstriated continuous myosin fibers in the cardiomyocytes in early days of culture. These fibers were described as having "...a stress fiberlike morphology...." In older cultures, the authors recorded typical myofibrils with ABands. They concluded that myofibrils could "... reassemble via the stress fiberlike intermediates in cultured cardiac myocytes."

A nonmuscle isoform of myosin II was first shown to be present in skeletal muscle cells near the membrane in fibers distinct from the mature myofibrils [30]. These fibers were later termed "stress-fiber-like" by Dlugosz et al. [31]. Three isoforms of nonmuscle myosin II have since been detected in fibers in vertebrate striated muscle cells [3234]. Subsequent studies have differed over the role that these "stress-fiber-like" fibers might play in myofibrillogenesis. They were suggested by the Holtzer lab to function as templates along which muscle proteins assemble and form cross-striated myofibrils [31]. The templates were thought to then disassemble with the subunits reassembling in another region of the cell to restart the templating process. Thus, one stress fiber-like structure was proposed to serve as the template for each new myofibril myofibril. 


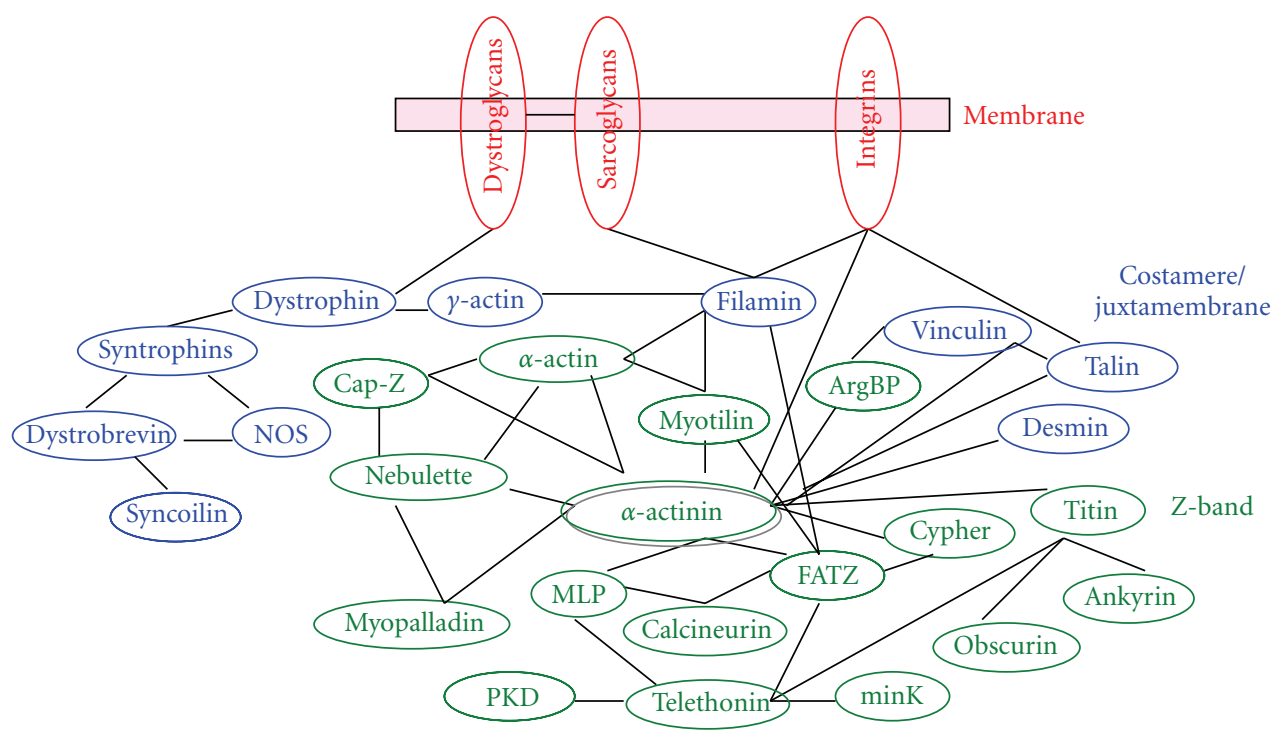

FIGURE 2: Diagram of some proteins reported to be in the Z-bands of mature myofibrils. The Z-bands of the mature myofibrils are attached via costameric proteins to the membrane of the muscle cells.

The fibers, that were nonstriated when F-actin were stained with phalloidin, were revealed to be formed of minisarcomeres when fluorescent alpha-actinin was injected into live cells or perfused into permeabilized muscle cells [3537]. Small puncta of alpha-actinin were spaced at intervals varying from 0.3 to 1.5 microns. In live cells injected with fluorescent alpha-actinin, the distance between puncta increased with time [37], as a later study confirmed in embryonic heart cells transfected with GFP-alpha-actinin in which small puncta of alpha-actinin aligned laterally and Zbands appeared beaded before appearing as a smooth band [38]. Bands of nonmuscle myosin II were detected alternating with the sarcomeric alpha-actinin densities (z-bodies) in these fibers [39]. Identical minisarcomeric patterns of alternating z-bodies (sarcomeric alpha-actinin) and bands of nonmuscle myosin II were also discovered in precardiac chick mesoderm explants [5] and in stained intact hearts in early chick embryos [40]. Thus there were no differences between cultured or intact hearts in the detection of a pathway for myofibrillogenesis (Figure 1). We suggest that myofibrillogenesis is a fundamentally conserved process, comparable to protein synthesis, mitosis, or cytokinesis, whether examined in situ or in vitro.

Nonmuscle myosin II light chains that were fluorescently labeled and injected into cultured cells from avian embryonic muscle also were localized in small periodically spaced bands along the thinnest actin fibers near the cell membrane [27]. In addition, the light chains also localized in A-bands in the myofibrils in the embryonic cells and in an unstriated pattern along the length of other actin fibers [27]. The unstriated pattern represents the nascent myofibrils (Figure 1). The unstriated pattern results from the overlapping thick filaments in the nascent myofibrils [5-7]. In dividing avian cardiomyocytes, Conrad et al. [41] discovered that nonmuscle myosin II, but not muscle myosin
II, was present in the cleavage furrows. The nonmuscle myosin II in avian cardiomyocytes is now known to be the II B isoform $[39,42]$. The antibody to II A does not react with embryonic chick cardiomyocytes [39], and early reports revealed that fibers with minisarcomeric bands of nonmuscle myosin were not present in avian cardiomyocytes $[43,44])$ resulted because antibodies to II A were used. The small diameter and unstriated appearance of the fibers with phalloidin staining fibers resembles those of stress fibers, thus the term stress fiber-like structures [31]. However, we now know that most of the proteins in these fibers are muscle-specific proteins, for example, alpha-actinin, actin, tropomyosin, and so forth $[7,39,45,46]$. They are also less sensitive than stress fibers to a number of different inhibitors (gelsolin [47], DNase1, Vitamin-D binding protein [48], and latrunculin-A [49]). J. M. Sanger and J. W. Sanger [50] demonstrated that cleavage furrows contained oppositely polarized actin filaments interdigitating with nonmuscle IIlike filaments in minisarcomeric patterns similar to those in the stress fibers of interphase cells.

We have proposed that the fibers with minisarcomeric bands of nonmuscle myosin II in muscle cells are premyofibrils, the precursors of myofibrils (see [7, 39]; Figure 1). We consider the small puncta of alpha-actinin $z$-bodies, precursors of Z-bands. Patterns of myosin II localization in embryonic chick cardiomyocytes fixed at different times after spreading and reformation of myofibrils in culture points to three types of fibers (Figure 1). Premyofibrils consist of thin filaments with periodic mini-A-Bands spaced between the puncta of alpha-actinin (z-bodies). Nascent myofibrils possess two types of myosin II staining: small periodic mini-A-Bands of nonmuscle myosin IIB and unbanded staining of muscle-specific myosin II. Mature myofibrils stain for only one type of myosin II, that is, the musclespecific myosin IIs in A-bands. Thus, the original unbanded 
staining of fibers with phalloidin, which Dlugosz et al. [31] originally termed stress fiber-like fibers, represents both pre- and nascent myofibrils. Subsequent observations of forming muscle in fixed embryonic avian myocytes by the Holtzer group led them to propose an additional model in which myofibril assembly occurred through independent assembly of thick filaments and Z-bands linked to their associated thin filaments (I-Z-I brushes) $[43,44]$. Titin was proposed to associate with the I-Z-I brushes, capture the muscle myosin II filaments and align them into A-bands, and join the A-bands and I-Z-I brushes into sarcomere units without involvement of stress fiber-like structures. However, it is now known that the precursors of the Zbands (z-bodies) and associated actin are not scattered in the cell but are aligned by the minisarcomeric arrangement of nonmuscle myosin IIs, that is, premyofibrils (Figure 1; $[7,39,51])$. The minisarcomeric arrangements of cleavage furrows, stress fibers, and premyofibrils would appear to have evolved very early $[27,28,50,52,53]$. Live cell imaging has allowed us to follow myofibrillogenesis from the deposition of premyofibrils to nascent myofibrils to mature myofibrils in cultured cardiomyocytes, skeletal muscle cells and in living zebrafish [37, 38, 49, 53-56]. Live cell imaging of muscle cells transfected with truncated Z-band regions of titin demonstrated the importance of titin to myofibrillogenesis $[57,58]$. We were able to use exposure of early myoblasts to different concentrations of ethyl methanesulfonate to arrest myofibrillogenesis at the premyofibril or nascent myofibril stage [55]. Removal of this inhibitor led to the resumption of the assembly process that resulted in mature myofibrils (Figure 1). The role of nonmuscle myosin II appears to be essential for the alignment of the thin filaments in the premyofibrils and nascent myofibrils $[5,6,49]$. Disruption of the formation of nonmuscle myosin II filaments with an inhibitor of the phosphorylation of the myosin light chains that are essential for the assembly of the filaments, leads to the loss of the premyofibrils and an unorganized array of myosin thick filaments [5]. Removal of the ML7 inhibitor led to the reformation of premyofibrils and nascent myofibrils and the assembly of ordered arrays of thick filaments in the A-bands in the mature myofibrils [5].

How the overlapping thick filaments composed of muscle myosin II hexamers in the nascent myofibrils become aligned into A-bands of mature myofibrils is not clear. Titin, Mband proteins, and other proteins like obscurin have been proposed to play roles in this process [59-62]. There are myofibrils that lack M-bands and yet their A-Bands are fully aligned. Lange et al. [63] have recently reported that A-Bands and myofibrils are formed normally in obscuring knockout mice. They did find that the longitudinal arrays of the sarcoplasmic reticulum (SR) were changed, supporting a role for coupling the SR to the myofibril.

The premyofibril model (Figure 1) has not been accepted universally because it appears discordant with several published papers (see review by Sanger et al. [7]). In particular one of two reports from the Adelstein lab revealed that mice null for nonmuscle myosin IIB [64], if they survived to birth, had abnormal hearts and brains and died on the day of birth. Nevertheless, the abnormal hearts did contain normal myofibrils suggesting that nonmuscle myosin IIB was not necessary for the formation of all myofibrils. Western gel studies in the few surviving animals, however, indicated that in half the surviving neonates, the myosin IIA isoform was upregulated, and thus the IIA isoform may have taken the place of the IIB isoform. Embryonic skeletal muscle cells have both isoforms of nonmuscle myosin IIA and IIB. Thus, the skeletal muscle cells were normal in these IIB knockouts, presumably due to the IIA being present. A recent paper by $\mathrm{Lu}$ et al. [34] reports that both isoforms of nonmuscle myosin IIs, A and B, are present in the early mouse hearts. In another paper the Adelstein group has reported that nonmuscle myosin II is a Z-band protein in both cardiac (IIB) and skeletal (A and B) muscle cells [65]. They also reported in the same paper that the IIB isoform was localized in the intercalated discs of fixed and stained cardiac muscle cells. Since these two studies from the Adelstein labs were published, a third isoform of nonmuscle myosin IIC has been discovered $[32,33]$. This isoform is also present in the heart, skeletal muscles, and several organs. The role of nonmuscle myosin IIC in myofibrillogenesis has still to be explored. Our use of antibodies directed against nonmuscle II A or B in cardiac and skeletal muscle cells has never detected these antigens in either Z-bands or intercalated discs $[5,39,40,56]$. Our preliminary results show that cardiac and skeletal muscle cells cotransfected with Cerulean Fluorescent Protein (CeFP) and one of the three isoforms of nonmuscle myosin IIs (Green Fluorescent Protein (GFP)-nonmuscle myosin II A or B or C), contradicting the results of Takeda et al. [65] paper. GFP-nonmuscle myosin IIs were not localized in the Z-bands of either type of muscle cells or in the intercalated discs of cardiac muscle cells. The three nonmuscle myosin II isoforms were localized in areas of the cells in linear arrays, in alternating arrays with z-bodies containing CeFP-alphaactinin, that is, premyofibrils.

There are two other papers that have been cited as inconsistent with the premyofibril model of myofibrillogenesis. Ehler et al. [59] and Rudy et al. [66] reported their inability to detect the presence of premyofibrils or nascent myofibrils in either intact fixed embryonic avian hearts or precardiac mesoderm explants. However, improved fixation and immunofluorescent techniques lead to the detection of nonmuscle myosin II B in organized arrays in both types of preparations $[5,40]$ that were identical to the premyofibrils and nascent myofibrils originally reported in cultured embryonic cardiomyocytes [39]. Furthermore, Du et al. [5] were able to reversibly inhibit the assembly of mature myofibrils by the application of ML-7, an inhibitor of myosin light chain kinase, an enzyme that is responsible for the phosphorylation of the nonmuscle light chains, and a prerequisite for the ability of nonmuscle myosin IIs to form filaments. Removal of this inhibitor led to the reformation of the premyofibrils with their arrays of miniA-bands of nonmuscle myosin II B and the resumption of myofibrillogenesis leading to the formation of mature myofibrils. All of these results support a common model for myofibrillogenesis in cardiomyocytes, whether in intact hearts, precardiac mesoderm explants, or in tissue culture as diagramed in Figure 1. The filaments of nonmuscle myosin 


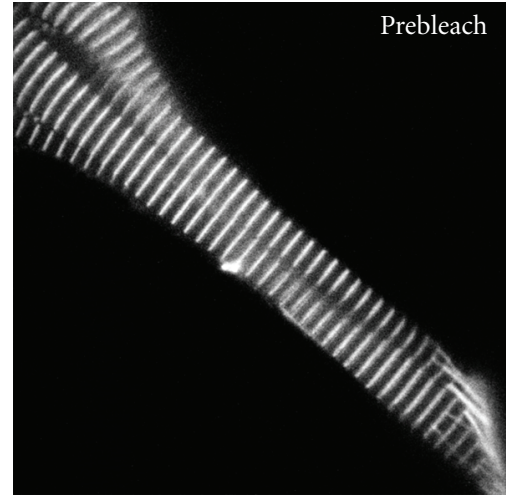

(a)

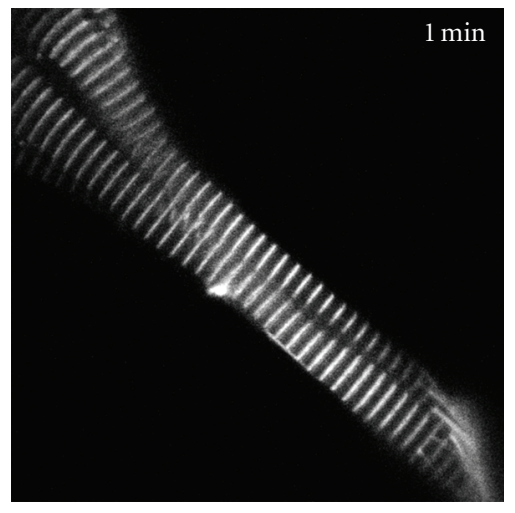

(c)

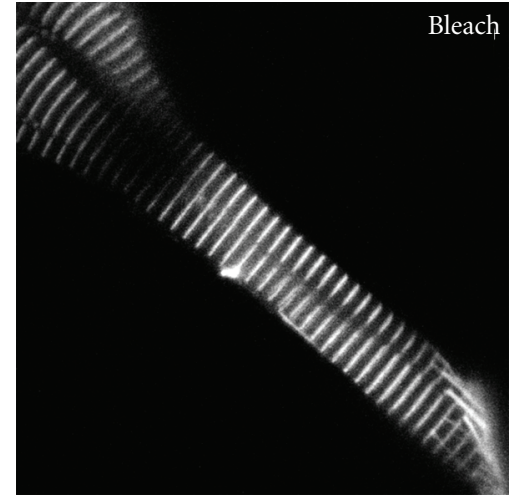

(b)

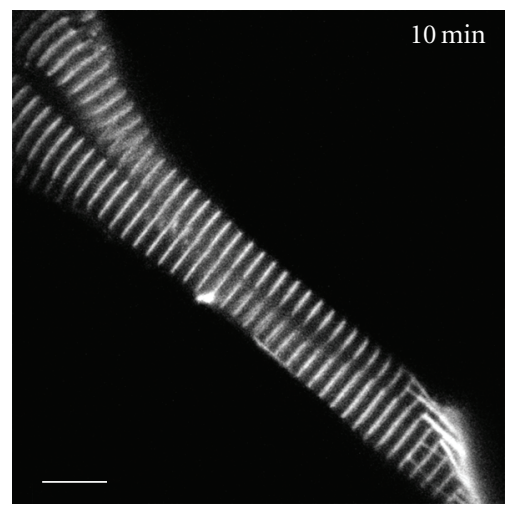

(d)

FIGURE 3: FRAP of YFP-myotilin in the Z-bands of mature myofibrils in two different muscle cells in a living zebrafish (three days old), prebleach, bleach, and 1 and 10 minutes after photobleaching. The recovery is almost complete by the end of the ten-minute period. Scale = 10 microns.

IIs may link the actin filaments attached to the z-bodies to form organized linear arrays we have termed premyofibrils $[7,39]$, the first step that leads to the formation of mature myofibrils (Figure 1).

\section{Optical Techniques to Explore Myofibrillogenesis}

Fluorescence microscopy of fluorescently tagged sarcomeric proteins (dye or GFP labels) has permitted myofibril assembly to be detected in living cardiac and skeletal muscle cells [37, 38, 49]. The technique of Fluorescence Recovery After Photobleaching (FRAP) has shown that superimposition on the addition of newly synthesized proteins to z-body and Zband complexes is an active exchange between molecules in a cytoplasmic pool and the same species of molecule residing in z-bodies and Z-bands [46, 56, 67-69]. In general, the extent and half time of the exchange of fluorescent molecules analyzed with FRAP techniques are a function of the binding interaction of the protein in the cellular complex where it is concentrated, with shorter recovery times suggesting lower affinity [70]. Wang et al. [46] demonstrated that seven different Z-band proteins exchanged independently of each other and that the exchange was independent of protein synthesis and molecular weight. Furthermore, the dynamics of the same proteins in z-bodies of premyofibrils were decreased in the Z-bands of the mature myofibrils. The decrease was suggested to be due to the incorporation of additional proteins into the forming of Z-band (Figure 1; [46]). These observations were extended to skeletal muscle cells in living zebrafish [56] where five different Z-bands were followed (actin, alpha-actinin, FATZ, myotilin, and telethonin). Their order of exchange was similar to the exchange of the same proteins in cultured quail skeletal muscle cells indicating that the behavior in culture conditions was comparable to that in cells in the live animal. Figures 3 and 4 are examples of this exchange process in the skeletal muscle cells along the flank of zebrafish using YFP-myotilin. The premyofibril model with its postulated three steps for the formation of myofibrils suggests mechanisms for the repositioning of molecules as premyofibrils are transformed into mature myofibrils over a period of several hours $[7,38]$. The exchange of molecules into and out of the forming myofibrils would permit this transformation (Figure 1).

Although fluorescence microscopy is widely used for examining colocalization of proteins in cells, the resolution limit of visible light microscopy is on the order of $200 \mathrm{~nm}$, 


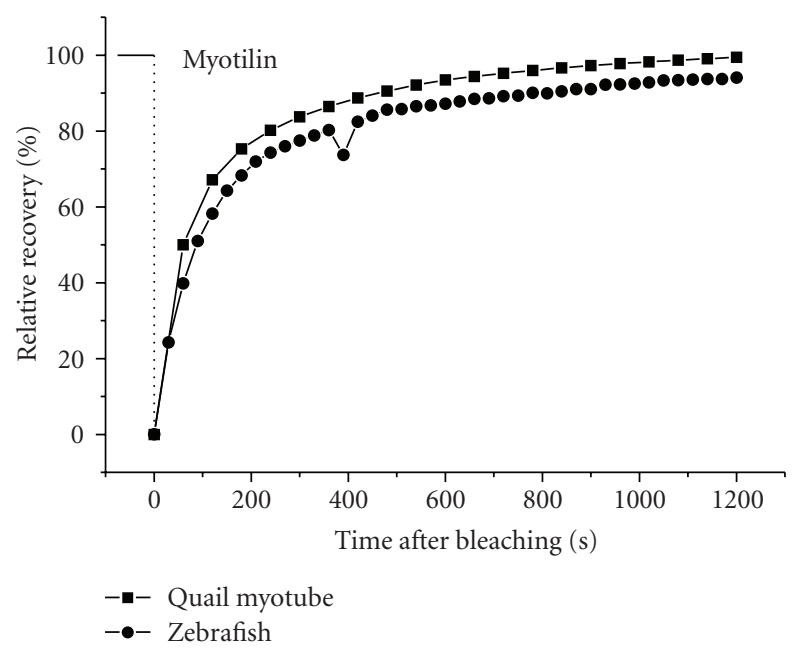

FIgURE 4: Comparison of the dynamics of YFP-myotilin after photobleaching in the Z-bands of mature myofibrils in skeletal muscle cells in tissue culture versus skeletal muscle cells along the flank of an embryonic zebrafish. Note the similarity of the recoveries of the same Z-band protein in the two different animals under two different conditions.

a distance much larger than the length scale of interprotein bonds. In the recent years, the technique of Fluorescence Resonance Energy Transfer (FRET) imaging has emerged as a way to improve upon the resolution of conventional light microscopy [10, 71]. FRET has been referred to as a "spectroscopic ruler" because it is possible, through application of the appropriate theory, to infer the distance between donor and acceptor from the resulting FRET signals in fixed and in living cells. Typically, measurable FRET signals result only when donor and acceptor are within $\sim 10 \mathrm{~nm}$ of each other. As fluorescence imaging technology has advanced, there has been growing interest in using FRET to map inter- and intramolecular interactions in cells. Stout et al. [8] used FRET techniques on living skeletal muscle cells to demonstrate that there are rearrangements of alpha-actinin and FATZ molecules as z-bodies fuse and realign to form Z-bands. These FRET results supported the interpretations of FRAP experiments during myofibrillogenesis [46] and the premyofibril model (Figure 1). The current development of microscopes that break the diffraction limit of light [72] should reveal further insights into the disposition of protein networks in myofibrils and the changes they undergo in the processes of assembly and disassembly.

In summary, our goal is to understand how myofibrils are assembled, remodeled, and maintained (Figures 1 and 3 ). We are using wild type and mutated sarcomeric proteins to investigate the assembly (confocal and deconvolution microscopes), dynamics and remodeling (FRAP), and interactions (FRET) of the proteins in myofibrils in the living state. Standard biochemical binding assays will be used to support newly revealed FRET relationships detected in living muscle cells. We will also be able to test the idea that myofibrillogenesis shares both the same pathway and molecular sarcomeric interactions, whether the assembly takes place in tissue culture or in a living animal like the zebrafish. In other words, we suggest that myofibrillogenesis is a fundamentally conserved process, comparable to protein synthesis, mitosis, or cytokinesis, whether examined in situ or in vitro.

\section{Acknowledgment}

This work was supported by NIH grants to Jean M. Sanger and Joseph W. Sanger.

\section{References}

[1] D. M. Chapman, C. F. A. Pantin, and E. A. Robson, "Muscle in coelenterates," Revue Canadienne de Biologie, vol. 21, pp. 267278, 1962.

[2] J. del Castillo, M. Anderson, and D. S. Smith, "Proventriculus of a marine annelid: muscle preparation with the longest recorded sarcomere," Proceedings of the National Academy of Sciences of the United States of America, vol. 69, no. 7, pp. 16691672, 1972.

[3] H. E. Huxley, "Electron microscope studies on the structure of natural and synthetic protein filaments from striated muscle," Journal of Molecular Biology, vol. 7, pp. 281-308, 1963.

[4] T. F. Robertson and S. Winegrad, "Variation of thin filament length in heart muscle," Nature, vol. 267, pp. 74-75, 1977.

[5] A. Du, J. M. Sanger, K. K. Linask, and J. W. Sanger, "Myofibrillogenesis in the first cardiomyocytes formed from isolated quail precardiac mesoderm," Developmental Biology, vol. 257, no. 2, pp. 382-394, 2003.

[6] J. W. Sanger, J. M. Sanger, and C. Franzini-Armstrong, "Assembly of the skeletal muscle cell," in Myology, A. G. Engel and C. Franzini-Armstrong, Eds., pp. 45-65, McGraw-Hill, New York, NY, USA, 3rd edition, 2004.

[7] J. W. Sanger, S. Kang, C. C. Siebrands, et al., "How to build a myofibril," Journal of Muscle Research and Cell Motility, vol. 26, no. 6-8, pp. 343-354, 2005.

[8] A. L. Stout, J. Wang, J. M. Sanger, and J. W. Sanger, “Tracking changes in Z-band organization during myofibrillogenesis with FRET imaging," Cell Motility and the Cytoskeleton, vol. 65, no. 5, pp. 353-367, 2008.

[9] S. Morimoto, "Sarcomeric proteins and inherited cardiomyocytes," Cardiovascular Research, vol. 77, pp. 659-666, 2008.

[10] D. W. Piston and G.-J. Kremers, "Fluorescent protein FRET: the good, the bad and the ugly," Trends in Biochemical Sciences, vol. 32, no. 9, pp. 407-414, 2007.

[11] K. A. Clark, A. S. McElhinny, M. C. Beckerle, and C. C. Gregorio, "Striated muscle cytoarchitecture: an intricate web of form and function," Annual Review of Cell and Developmental Biology, vol. 18, pp. 637-706, 2002.

[12] J. M. Sanger and J. W. Sanger, "The dynamic Z bands of striated muscle cells," Science Signaling, vol. 1, no. 32, article pe37, 2008.

[13] S. Y. Boateng and P. H. Goldspink, "Assembly and maintenance of the sarcomere night and day," Cardiovascular Research, vol. 77, no. 4, pp. 667-675, 2008.

[14] B. A. Danowski, K. Imanaka-Yoshida, J. M. Sanger, and J. W. Sanger, "Costameres are sites of force transmission to the substratum in adult rat cardiomyocytes," Journal of Cell Biology, vol. 118, no. 6, pp. 1411-1420, 1992. 
[15] J. M. Ervasti, "Costameres: the Achilles' heel of Herculean muscle," Journal of Biological Chemistry, vol. 278, no. 16, pp. 13591-13594, 2003.

[16] D. Frank, C. Kuhn, H. A. Katus, and N. Frey, "The sarcomeric Z-disc: a nodal point in signaling and disease," Journal of Molecular Medicine, vol. 84, pp. 446-468, 2006.

[17] S. Lange, E. Ehler, and M. Gautel, "From A to Z and back? Multicompartment proteins in the sarcomere," Trends in Cell Biology, vol. 16, no. 1, pp. 11-18, 2006.

[18] A. G. Engel and B. Q. Banker, "Ultrastructural changes in diseased muscle," in Myology, A. G. Engel and C. FranziniArmstrong, Eds., pp. 749-887, McGraw-Hill, New York, NY, USA, 3rd edition, 2004.

[19] M. Heidenhain, "Uber die Entstehung der quergestreiften Muskelsubstanz bei der Forelle," Archiv Mikroskopische Anatomie EntivMech, vol. 83, pp. 427-522, 1913.

[20] J. Auber, "La myofibrillogenese du muscle stile. I. lnsectes," Journal of Microscopy, vol. 8, pp. 197-232, 1969.

[21] J. Aronson, "Sarcomere size in developing muscles of a tarsonemid mite," The Journal of Biophysical and Biochemical Cytology, vol. 11, pp. 147-156, 1961.

[22] E. Lazarides and K. Weber, "Actin antibody: the specific visualization of actin filaments in nonmuscle cells," Proceedings of the National Academy of Sciences of the United States of America, vol. 71, pp. 2268-2271, 1974.

[23] J. W. Sanger, "Changing patterns of actin localization during cell division," Proceedings of the National Academy of Sciences of the United States of America, vol. 72, no. 5, pp. 1913-1916, 1975.

[24] E. Lazarides and K. Burridge, "Alpha-actinin: immunofluorescent localization of a muscle structural protein in non-muscle cells," Cell, vol. 6, pp. 289-299, 1975.

[25] K. Weber and U. Groeschel-Stewart, "Antibody to myosin: the specific visualization of myosin containing filaments in nonmuscle cells," Proceedings of the National Academy of Sciences of the United States of America, vol. 71, no. 11, pp. 4561-4564, 1974.

[26] J. W. Sanger, J. M. Sanger, and B. M. Jockusch, "Differences in the stress fibers between fibrblasts and epithelial cells," Journal of Cell Biology, vol. 96, no. 4, pp. 961-969, 1983.

[27] B. Mittal, J. M. Sanger, and J. W. Sanger, "Visualization of myosin in living cells," Journal of Cell Biology, vol. 105, no. 4, pp. 1753-1760, 1987.

[28] B. Mittal, J. M. Sanger, and J. W. Sanger, "Binding and distribution of fluorescently labeled filamin in permeabilized and living cells," Cell Motility and the Cytoskeleton, vol. 8, no. 4, pp. 345-359, 1987.

[29] R. R. Kulikowski and F. J. Manasek, "Myosin localization in cultured embryonic cardiac myocytes," in Motility in Cell Function, F. A. Pepe, J. W. Sanger, and V. T. Nachmias, Eds., pp. 433-435, Academic Press, New York, NY, USA, 1979.

[30] J. R. Fallon and V. T. Nachmias, "Localization of cytoplasmic and skeletal myosins in developing muscle cells by doublelabel immunofluorescence," Journal of Cell Biology, vol. 87, no. 1, pp. 237-247, 1980.

[31] A. A. Dlugosz, P. B. Antin, V. T. Nachmias, and H. Holtzer, "The relationship between stress fiber-like structures and nascent myofibrils in cultured cardiac myocytes," Journal of Cell Biology, vol. 99, no. 6, pp. 2268-2278, 1984.

[32] A. Leal, S. Endele, C. Stengel, et al., "A novel myosin heavy chain gene in human chromosome 19q13.3," Gene, vol. 312, no. 1-2, pp. 165-171, 2003.
[33] E. Golomb, X. Ma, S. S. Jana, et al., "Identification and characterization of nonmuscle myosin II-C, a new member of the myosin II family," Journal of Biological Chemistry, vol. 279, no. 4, pp. 2800-2808, 2004.

[34] W. Lu, S. H. Seeholzer, M. Han, et al., "Cellular nonmuscle myosins NMHC-IIA and NMHC-IIB and vertebrate heart looping," Developmental Dynamics, vol. 237, no. 12, pp. 35773590, 2008.

[35] J. W. Sanger, B. Mittal, and J. M. Sanger, "Formation of myofibrils in spreading chick cardiac myocytes," Cell Motility, vol. 4, no. 6, pp. 405-416, 1984.

[36] J. W. Sanger, B. Mittal, and J. M. Sanger, "Analysis of myofibrillar structure and assembly using fluorescently labeled contractile proteins," Journal of Cell Biology, vol. 98, no. 3, pp. 825-833, 1984.

[37] J. M. Sanger, B. Mittal, M. B. Pochapin, and J. W. Sanger, "Myofibrillogenesis in living cells microinjected with fluorescently labeled alpha-actinin," Journal of Cell Biology, vol. 102, no. 6, pp. 2053-2066, 1986.

[38] G. A. Dabiri, K. K. Turnacioglu, J. M. Sanger, and J. W. Sanger, "Myofibrillogenesis visualized in living embryonic cardiomyocytes," Proceedings of the National Academy of Sciences of the United States of America, vol. 94, no. 17, pp. 9493-9498, 1997.

[39] D. Rhee, J. M. Sanger, and J. W. Sanger, "The premyofibril: evidence for its role in myofibrillogenesis," Cell Motility and the Cytoskeleton, vol. 28, no. 1, pp. 1-24, 1994.

[40] A. Du, J. M. Sanger, and J. W. Sanger, "Cardiac myofibrillogenesis inside intact embryonic hearts," Developmental Biology, vol. 318, no. 2, pp. 236-246, 2008.

[41] A. H. Conrad, W. A. Clark, and G. W. Conrad, "Subcellular compartmentalization of myosin isoforms in embryonic chick heart ventricle myocytes during cytokinesis," Cell Motility and the Cytoskeleton, vol. 19, no. 3, pp. 189-206, 1991.

[42] A. H. Conrad, T. Jaffredo, and G. W. Conrad, "Differential localization of cytoplasmic myosin II isoforms a and B in avian interphase and dividing embryonic and immortalized cardiomyocytes and other cell types in vitro," Cell Motility and the Cytoskeleton, vol. 31, no. 2, pp. 93-112, 1995.

[43] M.-H. Lu, C. DiLullo, T. Schultheiss, et al., "The vinculin/sarcomeric- $\alpha$-actinin $/ \alpha$-actin nexus in cultured cardiac myocytes," Journal of Cell Biology, vol. 117, no. 5, pp. 1007-1022, 1992.

[44] H. Holtzer, T. Hijikata, Z. X. Lin, et al., "Independent assembly of $1.6 \mu \mathrm{m}$ long bipolar MHC filaments and I-Z-I bodies," Cell Structure and Function, vol. 22, no. 1, pp. 83-93, 1997.

[45] S. M. Wang, M. L. Greaser, E. Schultz, J. C. Bulinski, J. J. Lin, and J. L. Lessard, "Studies on cardiac myofibrillogenesis with antibodies to titin, actin, tropomyosin, and myosin," The Journal of Cell Biology, vol. 107, pp. 1075-1083, 1988.

[46] J. Wang, N. Shaner, B. Mittal, et al., "Dynamics of Zband based proteins in developing skeletal muscle cells," Cell Motility and the Cytoskeleton, vol. 61, no. 1, pp. 34-48, 2005.

[47] J. M. Sanger, B. Mittal, A. Wegner, B. M. Jockusch, and J. W. Sanger, "Differential response of stress fibers and myofibrils to gelsolin," European Journal of Cell Biology, vol. 43, no. 3, pp. 421-428, 1987.

[48] J. M. Sanger, G. Dabiri, B. Mittal, M. A. Kowalski, J. G. Haddad, and J. W. Sanger, "Disruption of microfilament organization in living non-muscle cells by microinjection of vitamin-D binding protein or DNaseI," Proceedings of the 
National Academy of Sciences of the United States of America, vol. 87, pp. 5474-5478, 1990.

[49] J. Wang, J. M. Sanger, and J. W. Sanger, "Differential effects of Latrunculin-A on myofibrils in cultures of skeletal muscle cells: insights into mechanisms of myofibrillogenesis," Cell Motility and the Cytoskeleton, vol. 62, no. 1, pp. 35-47, 2005.

[50] J. M. Sanger and J. W. Sanger, "Banding and polarity of actin filaments in interphase and cleaving cells," Journal of Cell Biology, vol. 86, no. 2, pp. 568-575, 1980.

[51] S. M. LoRusso, D. Rhee, J. M. Sanger, and J. W. Sanger, "Premyofibrils in spreading adult cardiomyocytes in tissue culture: evidence for reexpression of the embryonic program for myofibrillogenesis in adult cells," Cell Motility and the Cytoskeleton, vol. 37, no. 3, pp. 183-198, 1997.

[52] J. M. Sanger, J. S. Dome, and J. W. Sanger, "Unusual cleavage furrows in vertebrate tissue culture cells: insights into the mechanisms of cytokinesis," Cell Motility and the Cytoskeleton, vol. 39, no. 2, pp. 95-106, 1998.

[53] J. M. Sanger and J. W. Sanger, "Assembly of cytoskeletal proteins into cleavage furrows of tissue culture cells," Microscopy Research and Technique, vol. 49, no. 2, pp. 190-201, 2000.

[54] J. W. Sanger and J. M. Sanger, "Green fluorescent proteins improve myofibril research," Biophotonics International, vol. 8, no. 3, pp. 44-46, 2001.

[55] M. L. Golson, J. M. Sanger, and J. W. Sanger, "Inhibitors arrest myofibrillogenesis in skeletal muscle cells at early stages of assembly," Cell Motility and the Cytoskeleton, vol. 59, no. 1, pp. $1-16,2004$.

[56] J. W. Sanger, J. Wang, B. Holloway, A. Du, and J. M. Sanger, "Myofibrillogenesis in skeletal muscle cells in zebrafish," Cell Motility and the Cytoskeleton, vol. 66, no. 8, pp. 556-566, 2009.

[57] K. K. Turnacioglu, B. Mittal, G. A. Dabiri, J. M. Sanger, and J. W. Sanger, "Zeugmatin is part of the Z-band targeting region of titin," Cell Structure and Function, vol. 22, no. 1, pp. 73-82, 1997.

[58] K. K. Turnacioglu, B. Mittal, G. A. Dabiri, J. M. Sanger, and J. W. Sanger, "An N-terminal fragment of titin coupled to green fluorescent protein localizes to the Z-bands in living muscle cells: overexpression leads to myofibril disassembly," Molecular Biology of the Cell, vol. 8, no. 4, pp. 705-717, 1997.

[59] E. Ehler, B. M. Rothen, S. P. Hämmerle, M. Komiyama, and J.-C. Perriard, "Myofibrillogenesis in the developing chicken heart: assembly of Z-disk, M-line and the thick filaments," Journal of Cell Science, vol. 112, no. 10, pp. 1529-1539, 1999.

[60] P. F. M. Van der Ven, J. W. Bartsch, M. Gautel, H. Jockusch, and D. O. Fürst, "A functional knock-out of titin results in defective myofibril assembly," Journal of Cell Science, vol. 113, no. 8, pp. 1405-1414, 2000.

[61] M. Gotthardt, R. E. Hammer, N. Hübner, et al., "Conditional expression of mutant M-line titins results in cardiomyopathy with altered sarcomere structure," Journal of Biological Chemistry, vol. 278, no. 8, pp. 6059-6065, 2003.

[62] A. Kontrogianni-Konstantopoulos, D. H. Catino, J. C. Strong, and R. J. Bloch, "De novo myofibrillogenesis in C2C12 cells: evidence for the independent assembly of $\mathrm{M}$ bands and $\mathrm{Z}$ disks," American Journal of Physiology, vol. 290, no. 2, pp. C626-C637, 2006.

[63] S. Lange, K. Ouyang, G. Meyer, et al., "Obscurin determines the architecture of the longitudinal sarcoplasmic reticulum," Journal of Cell Science, vol. 122, no. 15, pp. 2640-2650, 2009.

[64] A. N. Tullio, D. Accili, V. J. Ferrans, et al., "Nonmuscle myosin II-B is required for normal development of the mouse heart," Proceedings of the National Academy of Sciences of the United States of America, vol. 94, no. 23, pp. 12407-12412, 1997.
[65] K. Takeda, Z.-X. Yu, S. Qian, T. K. Chin, R. S. Adelstein, and V. J. Ferrans, "Nonmuscle myosin II localizes to the Z-lines and intercalated discs of cardiac muscle and to the Z-lines of skeletal muscle," Cell Motility and the Cytoskeleton, vol. 46, no. 1, pp. 59-68, 2000.

[66] D. E. Rudy, T. A. Yatskievych, P. B. Antin, and C. C. Gregorio, "Assembly of thick, thin, and titin filaments in chick precardiac explants," Developmental Dynamics, vol. 221, no. 1, pp. 61-71, 2001.

[67] N. M. McKenna, J. B. Meigs, and Y.-L. Wang, "Exchangeability of alpha-actinin in living cardiac fibroblasts and muscle cells," Journal of Cell Biology, vol. 101, no. 6, pp. 2223-2232, 1985.

[68] J. Wang, J. M. Sanger, S. Kang, et al., "Ectopic expression and dynamics of TPM $1 \alpha$ and TPM $1 \kappa$ in myofibrils of avian myotubes," Cell Motility and the Cytoskeleton, vol. 64, no. 10, pp. 767-776, 2007.

[69] J. Wang, H. Thurston, E. Essandoh, et al., "Tropomyosin expression and dynamics in developing avian embryonic muscles," Cell Motility and the Cytoskeleton, vol. 65, no. 5, pp. 379-392, 2008.

[70] M. Vink, M. Simonetta, P. Transidico, et al., "In vitro FRAP identifies the minimal requirements for Mad2 kinetochore dynamics," Current Biology, vol. 16, no. 8, pp. 755-766, 2006.

[71] L. Stryer, "Fluorescence energy transfer as a spectroscopic ruler," Annual Review of Biochemistry, vol. 47, pp. 819-846, 1978.

[72] K. R. Chi, "Microscopy: ever-increasing resolution," Nature, vol. 462, no. 7273, pp. 675-678, 2009. 

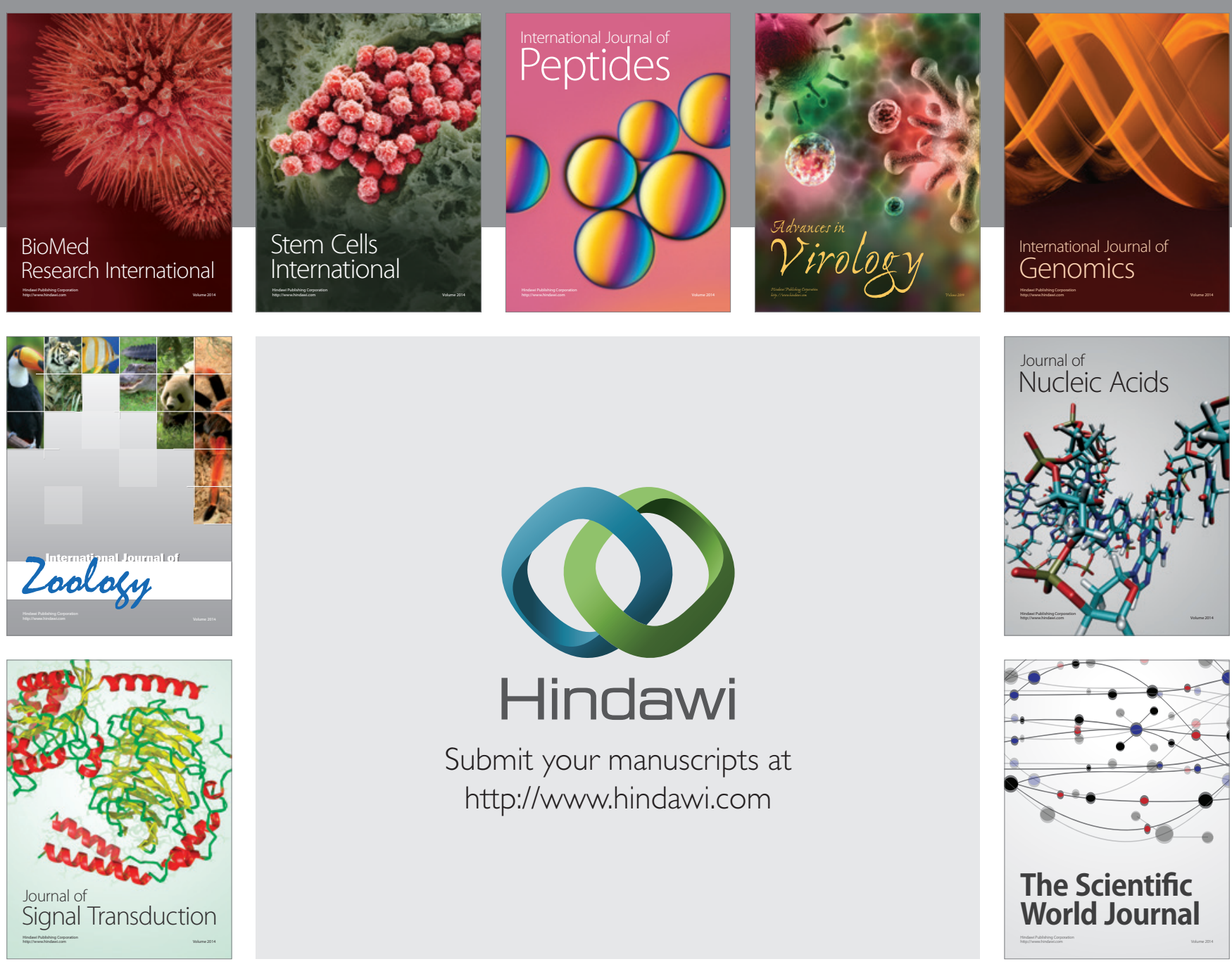

Submit your manuscripts at

http://www.hindawi.com
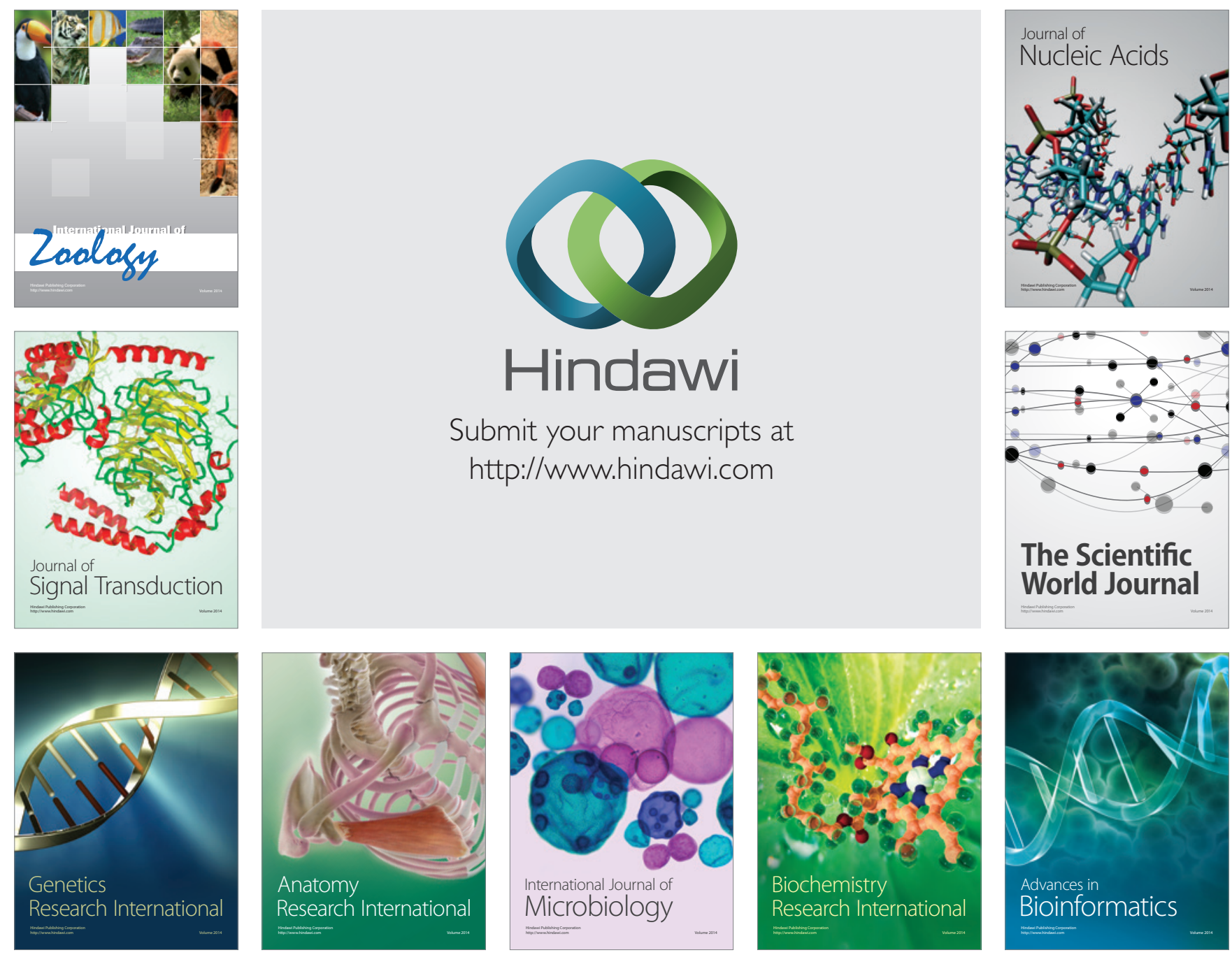

The Scientific World Journal
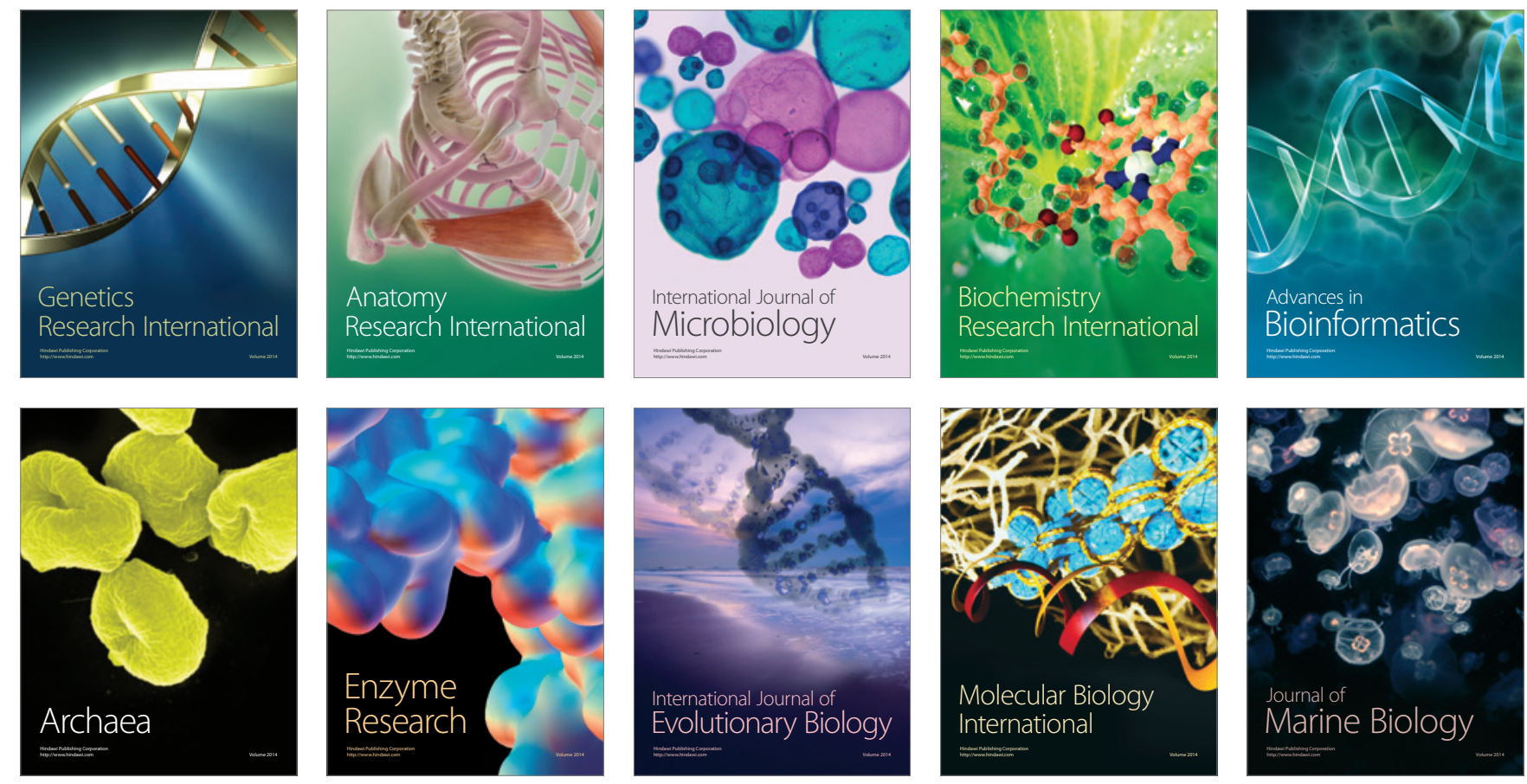